\title{
INFLUENTIAL FACTORS AFFECTING MATERIALS MANAGEMENT IN CONSTRUCTION PROJECTS
}

\author{
Zairra Mat Jusoh, Narimah Kasim \\ Onn Malaysia, Malaysia \\ Corresponding author: \\ Narimah Kasim \\ Faculty of Technology Management and Business \\ Universiti Tun Hussein Onn Malaysia \\ 81400 Parit Raja, Johor, Malaysia \\ phone: (+607) 4533841 \\ e-mail:narimah@uthm.edu.my
}

Department of Construction Management, Faculty of Technology Management and Business, Universiti Tun Hussein

Received: 30 March 2016 Accepted: 3 August 2017

\begin{abstract}
Construction projects are more often than not plagued by poor performances such as delays cost overrun, low productivity, construction wastes and compromised quality. Amongst the critical contributory factors of poor project performances, is the ineffectiveness of materials management occurring in the construction sites. Indeed, materials management is a very important component for construction projects. However, there are only limited numbers of research available regarding this topic. Thus, this research focuses its study on materials management, specifically in identifying the influential factors that affect materials management in the construction project activities. Literatures from books, journal articles and conference papers related to poor project performances and materials management have been reviewed. Consequently, this study sorted the salient influential factors and categorized them based on their specific group. Out of 47 factors identified, they are classified into 8 groups. They are (1) site condition; (2) planning and handling on site; (3) management; (4) materials; (5) supplier and manufacturer default; (6) transportation; (7) contractual; and (8) governmental interferences. In conclusion, this study contends that by identifying the influential factors affecting materials management, it will help construction players to avoid the occurrence of those factors and will minimize the negative impacts on the overall performance of construction projects. Hence, the handling-over of project will be according to schedule and not delayed by materials mismanagement.
\end{abstract}

KEYWORDS

construction projects, project performance, materials management, influential factors.

\section{Introduction}

Construction industry plays a vital role in the socioeconomic growth of a country and is an agent for national development $[1,2]$. Economically, it generates increase in the nation's gross domestic product (GDP) $[3,4]$ and contribute significantly to the national wealth and economic prosperity. Additionally, construction projects also play a significant role in safety, health and environmental aspects of the society by creating physical facilities and social infrastructure $[1,5]$. In Malaysia, the construction industry has been an important and productive sector as it contributes significantly to the economic growth with $3-5$ per cent of the national economy GDP $[5,6]$. In addition, the Tenth Malaysia Plan (2011-2015) has recognized that this industry had a great impact on the Malaysian construction sector. Hence, the Ministry of Works had been allocated with RM138 billion for its physical development projects to be undertaken by construction sector under this plan [7]. This shows that the contribution of this sector is essential to the national economy and has an important role in improving the people's quality of life $[5,8]$.

However, despite the importance of the construction industry to the nation's socioeconomic growth, this industry faces many challenges and issues relat- 
ed to poor construction project performances $[9,10]$. This poor performance has been attributed to the ineffective resources management in the construction projects $[11,12]$. As a result, construction projects had suffered with delays, cost overrun, construction wastes, low productivity, compromised quality etc. [1, 8, 12-16]. Prior literature reports that shortage of materials is among the most significant factors that contribute to delays, cost overrun and low productivity $[14,17,18]$ Therefore this again shows that ineffective materials management that causes materials shortage occurs in construction sites affects project performance [19].

However, literature also reveals that very little research has been carried out by academics and practitioners on the problems faced by the local construction industry [1]. This is specifically in terms of local construction materials management effectiveness [20-22]. Nonetheless topics regarding materials management practices and information and communication technology (ICT) have been researched before. But, there are limited studies that focus on influential factors affecting materials management. As it is essential to gain better insights and understanding of the factors influencing project performance, this study is now being embarked. Therefore, this study aims to identify the influential factors affecting materials management in construction projects and then to highlight the identified influential factors.

\section{Materials management in construction projects}

Construction materials constitute a major portion of the total cost in a construction project. It has been pointed out that the construction materials can constitute 50-60 per cent of the project cost [18]. Due to its role and major portion in construction projects, materials management has becomes a critical component and an essential function that improves productivity in construction projects. Thus, it is necessary for construction companies to understand the effects of proper materials management towards the success of project execution [19].

Materials management is defined by Business Roundtable in Modern Management System as 'the planning and controlling of all necessary efforts to ensure that the correct quality and quantity of materials and equipment are appropriately specified in a timely manner are obtained at a reasonable cost, and are available when needed' [19, 23]. However, some defined it directly with a concept that involved integrating process whereby all procurements of materials are combined under one management func- tion $[24,25]$. Briefly, materials management can be defined as a planned process that consist of identifying, purchasing, delivering, handling, allocating of storage and minimizing wastes with the purpose of ensuring the availability of sufficient quantities of material for project needs [19, 21].

Materials management in a construction project consists of seven integrated processes and functions $[19,23]$. They include project planning, materials take-off, suppliers' enquiry, purchasing, materials control, warehousing, expediting and shipping.

\section{Project planning}

In materials management function, the planning phase starts with identification and determination of required materials that need to be purchased and its availability [19, 26, 27]. This function covers scheduling and logistic planning as it is important to ensure an efficient transfer of materials from the suppliers to the project site in a timely manner and can fulfil the construction need [21-28].

\section{Materials take-off}

Materials take-off function is to acquire an actual total quantity of materials used in the construction projects. This process is able to identify the materials needed, including its specifications and calculate the required quantity of materials [23].

\section{Suppliers enquiry}

This process involves the identification and selection of suppliers. The criteria that need to be considered during the selection of suppliers are materials quality, delivery dependability, materials price, suppliers' service and performance [19, 23, 25].

\section{Purchasing}

Primarily, materials management process is associated with the purchasing process. This process is associated with the purchase of materials outside the organization to support the companies' operations and the materials must be purchased in a timely manner to ensure the availability of materials in the construction sites [19, 23].

\section{Materials control}

Materials control function includes materials handling, distribution and usage [29, 30]. This function is important to avoid any potential materials shortage or surplus occurring at the construction sites. The efficiency of this function optimizes the construction productivity and minimizes the construction wastes $[23,31]$. 


\section{Warehousing}

Warehousing relates to materials storage and it is important because the duration between ordering and receiving materials is uncertain. Therefore, there is a necessity that delivered materials need to be stored within the site area [30].

\section{Expediting and shipping}

This process is to ensure that suppliers deliver materials, technical specification, and equipment to the construction sites as per schedule based on the terms, stipulated date in the purchase order and the project's requirements and schedules. The purpose of expediting is to provide timely information related to materials deliveries [19, 23].

\section{Influential factors affecting materials management}

The factors influencing materials management have been the subject of inquiry especially in aspects such as time overrun, cost overrun, construction wastes and low productivity. However, studies that focused and discussed solely on factors of materials management is very limited. Furthermore, in order to improve project performance, it is necessary to identify the factors affecting materials management. No doubt there have been several researchers who have identified the influential factors under numerous topic. Studies done in at least four countries showed certain factors influenced materials management. For example a study in the construction labour productivity in Turkey revealed that materials management is a very important factor that impacted labor productivity. The factors associated with materials management were fluctuations in material prices, extensive multiple-handling of materials, improperly sorted or marked materials that made them difficult to define, wrong materials from the specification, poor quality due to production errors, unsystematic flow of material, improper material usage, improper planning, design changes leaving management little time to order necessary materials, on-site transportation and congested site [32].

Similarly, a study on factors influencing craftsmen's productivity in Chile reported that the main identified factor was materials-related problem which consist of 'lack of on-time delivery', 'insufficient equipment to move materials' or 'lacking of onsite logistic' and 'excessive paperwork' [17]. Moreover, another study in Indonesia also has reported a similar finding that 'lack of materials' has affected the craftsmen's productivity. The causes of nonavailability of materials were due to on-site transportation difficulties, excessive paper works, improper materials storage and inadequate planning [33]. Generally, the availability of materials on construction sites has major implications to construction labours' productivity. On the contrary, a study conducted in Australia revealed that lack of materials was ranked fifth as having an effect on the construction productivity. Other factors that contributed to lack of materials were shortage of fund, inadequate planning, excessive paperwork, improper materials usage with respect to specifications, fluctuation in materials availability, waste due to negligence, improper materials storage, poor delivery of materials to site, on-site transportation difficulties and poor materials handling on-site [4]. Even though the findings showed that 'lack of materials' was ranked at fifth place, the ineffective materials management still contributed to poor productivity in the Australian construction projects.

The studies above had identified from the literature reviews, several factors that affect materials management. However, in an effort to identify the factors exhaustively, all the factors must relate to the process and function of materials management i.e. project planning, materials take-off, vendor enquiry, purchasing, materials control, warehousing, expediting and shipping. This means that the identified influential factors should contribute to the shortage of materials. It is noted also from the literature review that some of the factors are interrelated or overlapped with each other. Sometimes, the factor is termed differently, but it represents the same meaning. For example 'use of incorrect material' is similar to 'improper material usage' in meaning. Hence, in order to represent the influential factors in a suitable arrangement and to avoid duplication, this study has made some rationalization. The alteration either involves rephrasing or remains in the original phrase as highlighted by previous researchers.

In summary, a total of 47 influential factors have been initially identified from different studies by previous researchers. These factors have been identified from 28 published papers in various topics such as time overrun, cost overrun, construction wastes, low productivity as well as materials management. Table 1 shows the list of the 47 influential factors and their sources as per enlisted references (in brackets). The frequency column is useful to see the most mentioned factors in previous studies. 
Management and Production Engineering Review

\begin{tabular}{|c|c|c|c|c|c|c|c|c|c|c|c|c|c|c|c|c|c|c|c|c|c|}
\hline 象 & 10 & $\infty$ & 0. & $+\gamma$ & $\infty$ & 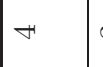 & $\infty$ & $r$ & $\sim$ & $\curvearrowright$ & $\sim$ & $\curvearrowright$ & -1 & -1 & $\infty$. & $\begin{array}{lll}0 & \infty\end{array}$ & $\forall$ & $\curvearrowright$ & $\curvearrowright$ & $\neg \curvearrowright$ & 0 \\
\hline if & & & & & $>$ & $>$ & & & & & & & & & & & & & & & \\
\hline is & & $>$ & & $>$ & & & $>$ & & & & & & & & & & & & & $>$ & $>$ \\
\hline 2 & $>$ & & & & & $>$ & & $>$ & & & & & & & & & & & & & \\
\hline : & & & & & & & & & & & & & & & $>$ & & & & & & \\
\hline $\bar{s}$ & & & & & $>$ & & & $>$ & & $>$ & & & & & & & & & $>$ & & $>$ \\
\hline $\bar{\alpha}$ & & $>$ & $>$ & & & & & & & & & & & & & $>$ & & & & & \\
\hline I & 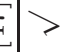 & & & & & & $>$ & $>$ & & & & & & & & & & & & & \\
\hline 乌 & & $>$ & $>$ & & & & & & & & $>$ & & & & & $>$ & & & & & \\
\hline 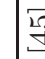 & & & & Y & $>$ & & & & & & & & & & & $>$ & $>>$ & & & & \\
\hline$\exists$ & & & $>$ & & $>>$ & $>$ & & & $>$ & & & & & & & & & & & $>$ & $>$ \\
\hline$\stackrel{\tilde{y}}{\mathrm{y}}$ & & & & & & & & & & & & & & & $>$ & & $>$ & & & & \\
\hline $\bar{\xi}$ & & & $>$ & & & & & & & & & $>$ & & & & & & & & & \\
\hline$\exists$ & & & & & $>$ & & & & & & & & & & $>1$ & $>$ & & & & & \\
\hline 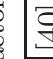 & $>$ & & & & & & & $>$ & $>$ & & & & & & & & & & & & \\
\hline త్ర & & $>$ & $>$ & & & & & & & & $>$ & $>$ & $>$ & $>$ & & & & $>$ & & $>$ & \\
\hline 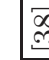 & & & & & & $>$ & & & & & & & & & $>$ & & & & & & \\
\hline 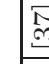 & & & & & & & & & & & & & & & & $>$ & & & & & \\
\hline 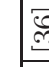 & & & & & & & & & & & & & & & $>$ & & & & & & $>$ \\
\hline 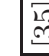 & & & & & & & & & & & & & & & & & $>$ & & & & \\
\hline 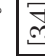 & & & & & & & & & & & & & & & & $>$ & & & & & $>$ \\
\hline 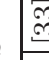 & & & & $>$ & & & & & & & & & & & & & & & & $>$ & \\
\hline জ্ & & $>$ & & & & & & & & & & & & & $>1$ & $>$ & & & & $>>$ & \\
\hline ฐ & $>$ & & & & & & & & & $>$ & & & & & & & & & $>$ & & $>$ \\
\hline I & & & & $>$ & & & & & & & & & & & & & & & & $>$ & $>$ \\
\hline$\exists$ & & & & 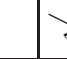 & $>$ & & & & & & & & & & & $>$ & & & & & \\
\hline$\overline{9}$ & & $>$ & $>$ & & & & & & & & & & & & $>$ & & & $>$ & & & $>$ \\
\hline$\overline{5}$ & $1>$ & $>$ & & $>$ & & & $>$ & & & & & & & & 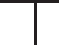 & & $>$ & 1 & & $>$ & $>$ \\
\hline$\pi$ & & $>$ & & & & & & & & & & & & & $>1$ & $>$ & & & & & \\
\hline$=$ & 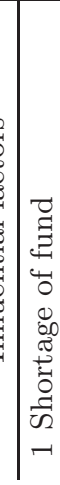 & 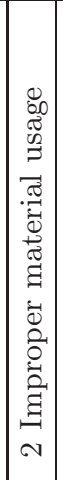 & 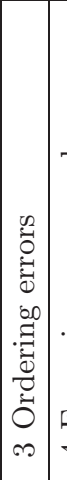 & 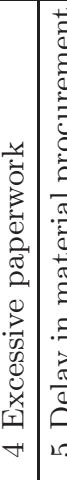 & 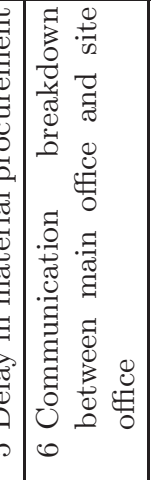 & 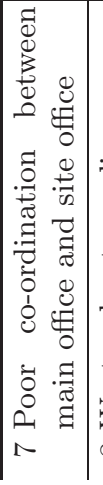 & 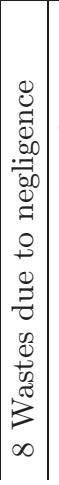 & 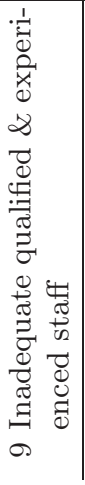 & 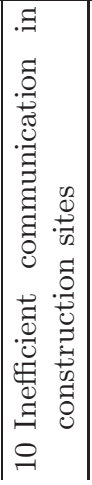 & 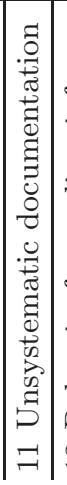 & 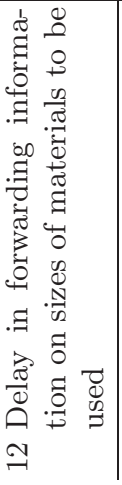 & 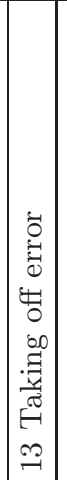 & 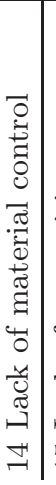 & 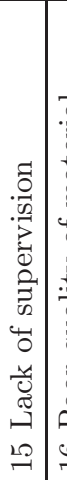 & 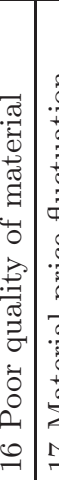 & 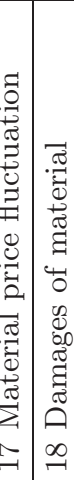 & 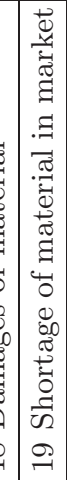 & 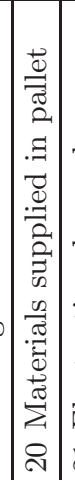 & 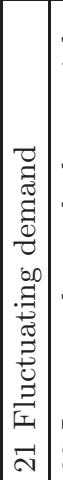 & 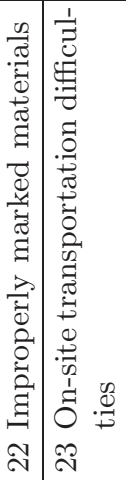 & 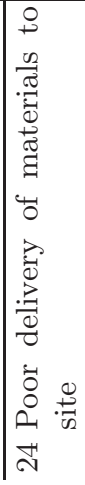 \\
\hline
\end{tabular}




\begin{tabular}{|c|c|c|c|c|c|c|c|c|c|c|c|c|c|c|c|c|c|c|c|c|c|c|c|}
\hline 离 & $\infty$ & $\exists$ & $\stackrel{0}{-1}$ & $\not$ & $\sim$ & $\neg$ & os & -1 & $\vec{F}$ & 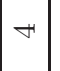 & -1 & - & 0 & $\infty$ & $\infty$ & - & -1 & - & $\forall$ & - & $\infty$ & - & - \\
\hline 䔲 & & & & & & & $>$ & & $>$ & & & & $>$ & & & & & & & & & & \\
\hline is & & $>$ & $>$ & & & & & & $>$ & & & & & & & & & & & & & & $>$ \\
\hline $\overrightarrow{20}$ & & & & & & & & & 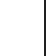 & & & & & & & & & & & & & & \\
\hline 웅 & & & & & & & & & . & & & & & & $>$ & & & & & & & & \\
\hline F্ & & $>$ & & & & & & & 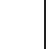 & & & & & & & & $>$ & $>$ & $>$ & & & $>$ & \\
\hline \begin{tabular}{|l|}
$\infty$ \\
$\Phi$ \\
\end{tabular} & & $>$ & & & & & & & $>$ & & & & & & & & & & $>$ & & & & \\
\hline 等 & & 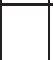 & & & & & $>$ & & 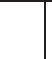 & & & & & & $>$ & & & & & & & & \\
\hline 果 & & $>$ & & & & & $>$ & & $>$ & & & & & & & & & & $>$ & & & & \\
\hline$\stackrel{8 g}{9}$ & & 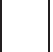 & & & & & & & 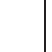 & & & & $>$ & & $>$ & & & & & & $>$ & & \\
\hline F & & & $>$ & & & & & & $>$ & $>$ & & & & $>$ & & & & & & & & & \\
\hline 粱 & & & $>$ & & & & & & $>$ & & & & & & & & & & & & & & \\
\hline$\underset{\mathcal{I}}{\mathcal{I}}$ & & & $>$ & & & & & & 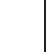 & $>$ & & & & & & & & & & & & & \\
\hline$\exists$ & & $>$ & & & & & & & 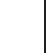 & & & & & & & & & & & & & & \\
\hline F & & $>$ & & & & & $>$ & & $>$ & & & & & & & $>1$ & & & & & & & \\
\hline \begin{tabular}{|l|}
$\vec{\rho}$ \\
\end{tabular} & $>$ & $>$ & $>$ & $>$ & $>$ & & $>$ & & 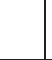 & $>$ & $>$ & $>$ & & $>$ & & & & & & & & & \\
\hline 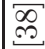 & $>$ & & $>$ & $>$ & $>$ & & & & & $>$ & & & $>$ & & & & & & & & & & \\
\hline 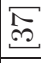 & & & & & & & & & & & & & & & & & & & & & & & \\
\hline$\ddot{\mathscr{c}}$ & & & & & & & & & & & & & & & & & & & & & & & \\
\hline 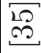 & & & $>$ & & & & & & $>$ & & & & & & & & & & & & & & \\
\hline Æु & & & & & & & $>$ & & $>$ & & & & & & & & & & & & & & \\
\hline אֶ, & & $>$ & & & & & & & $>$ & & & & & & & & & & & & & & \\
\hline ๙ָ๊ & & $>$ & $>$ & & & $>$ & $>$ & $>$ & . & & & & & & & & & & $>$ & & $>$ & & \\
\hline$\stackrel{乛}{\stackrel{2}{N}}$ & & & & $>$ & & & & & & & & & $>$ & & & & & & & & & & \\
\hline$\cong$ & & & & & & & & & & & & & & & & & & & & & & & \\
\hline$\nexists$ & & & & & & & $>$ & & $>$ & & & & $>$ & & & & & & & & $>$ & & \\
\hline $\bar{\Theta}$ & $>$ & & $>$ & $>$ & & & $>$ & $>$ & $>$ & & & & $>$ & $>$ & & & & & $>$ & & & & \\
\hline$F$ & & $>$ & $>$ & & & & & & $>$ & & & & & & & & & & & & & & \\
\hline$\overline{\mathrm{N}}$ & & $>$ & & & & & & & $>$ & & & & & & & & & & & $>$ & & & \\
\hline 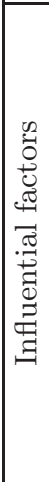 & 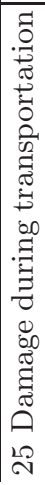 & 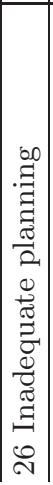 & 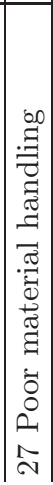 & 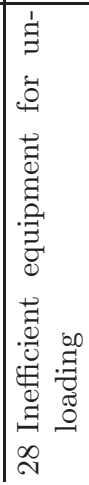 & 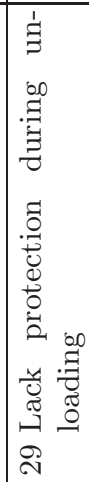 & 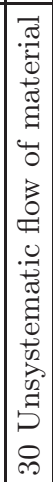 & 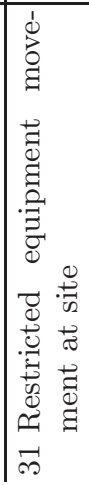 & 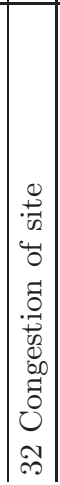 & 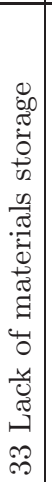 & 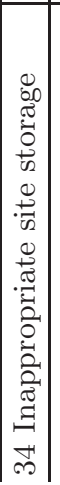 & 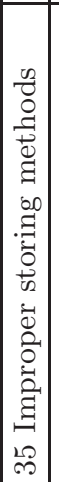 & 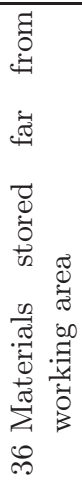 & 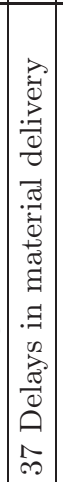 & 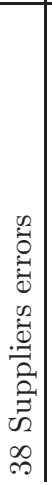 & 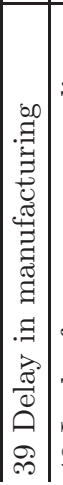 & 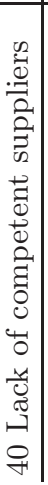 & 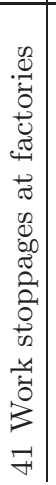 & 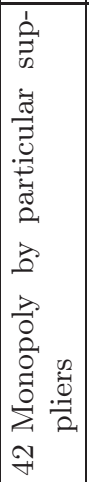 & 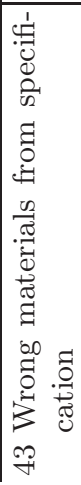 & 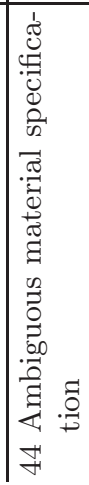 & 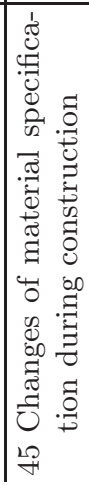 & 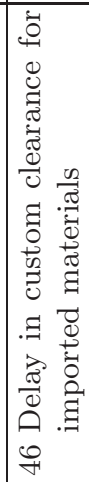 & 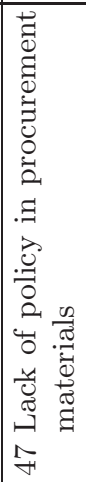 \\
\hline
\end{tabular}


In the mean time, several approaches have been adopted by this study in relation to classifying the influential factors group that affect materials management. However, based on previous studies, the classification schemes of each group is quite varied. Reference [43] classified the factors affecting labour productivity into 10 groups: manpower, leadership, motivation, time, material/tools, supervision, project, safety, quality and external factors. Another study sorted the factors contributing to construction wastes into 7 categories: design, handling, workers, management, site condition, procurement and external factors [13]. Interestingly, an author classifies the construction wastes based on the origin and causes [39]. The group classification proposed by the latter author is found relevant therefore acceptable to be adopted in this study. The identified influential factors are categorized into 8 groups: site condition, planning and handling on site, management, material, transportation, supplier or manufacturer default, contractual and governmental interferences. Figure 1 illustrates the Fish bone influential factors that are classified into respective 8 groups affecting materials management efficiency.

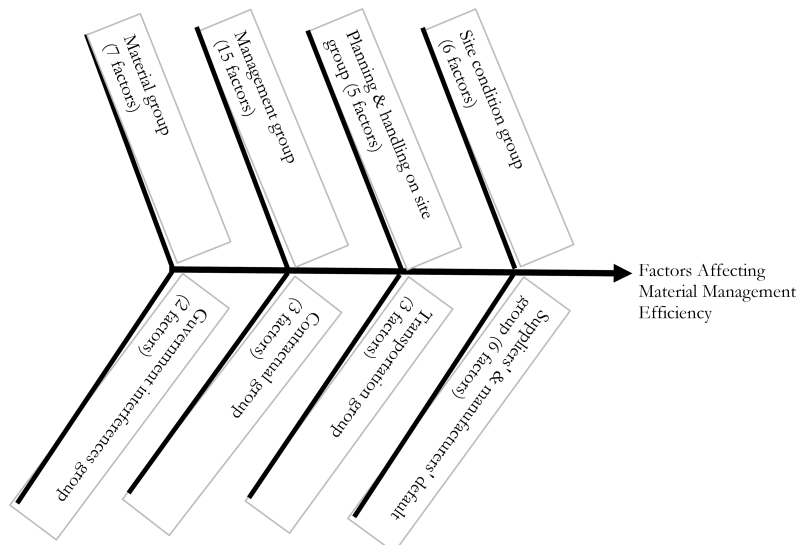

Fig. 1. Fish bone diagram of group factors affecting materials management efficiency [54].

\section{Site condition group}

The formation of the 'site condition' group is adapted from the classification proposed by previous author [13]. It has been agreed that it is needed to consider site condition while dealing with materials management [30]. In this category, there are 6 influential factors: lack of materials storage, restricted equipment movement at site, unsuitable site storage, improper storing methods, congestion of site and materials stored far from working area. Generally, under this category, the factors are related to access and storage on construction site.

\section{Planning and handling on site group}

The formation of the "planning and handling on site' category is based on the group classification proposed by previous authors $[14,48]$. This group consists of 5 influential factors: inadequate of planning, poor material handling, inefficient equipment for unloading, lack of protection during unloading and unsystematic flow of materials.

\section{Management group}

The formation for the 'management' group is adapted and modified from previous studies [39, 49]. This group comprises 15 influential factors: improper material usage, ordering errors, shortage of fund, excessive paperwork, delay in materials procurement, communication breakdown, poor coordination, waste due to negligence, lacking in qualified staffs, inefficient communication in construction sites, unsystematic documentation, delay in forwarding information on sizes of materials to be used, taking off error, lack of materials control and lack of supervision.

\section{Materials group}

The 'materials' group is adapted according to a previous study that classified the factors under the group as 'material and tool' [43]. However, in order for the factors to be more specific and related to materials, the group name proposed by previous study as 'material and tools' is now changed simply to 'materials' group. In this category, there are 7 influential factors: poor quality of materials, materials price fluctuation, damages of materials, shortage of materials in market, materials supplied without pallet, fluctuating demand of materials and improperly marked of materials.

\section{Suppliers and manufacturers' default group}

The formation of this group is based on the group proposed by a previous study that named it as the 'suppliers' default' [49]. After reviewing the identified influential factors, and in order to reasonably represent the influential factors under this group, the group name is expanded into 'suppliers' and manufacturers' default'. This group consists of 6 influential factors: delay in materials delivery by suppliers, suppliers errors, delay in manufacturing, lack of competent suppliers, work stoppages at factories and monopoly control by particular supplier.

\section{Transportation group}

The 'Transportation' group is classified according to what was proposed by previous studies [39, 49]. Hence, this group name which is 'transportation' is 
also applied to categorize the influential factors. This group consists of 3 influential factors: poor delivery of materials to site, on-site transportation difficulties and damage during transportation.

\section{Contractual group}

This group formation is based on the category proposed by a previous study that categorized it as 'lack of clarity in project scope' group [14]. However, the group name is rephrased into 'contractual' group to reasonably represent each influential factor. In this group, there are 3 influential factors: wrong materials from specification, changes of materials specification during construction and ambiguous materials specification.

\section{Governmental interference group}

The formation of this group name is based on a previous study [49]. The authors proposed the group name as 'governmental regulation'. However, in order to be generally more representative of the identified influential factors by the previous researchers, the group name is altered to 'governmental interference' group. This group only consists of 2 influential factors: delay in custom clearance for imported materials and lack of policy in procuring materials.

Overall, this study has extensively identified the various factors might affect materials management efficiency in construction projects. Since there is a wide array of factors involved, managing materials therefore become more complex and difficult [55]. It is found that the conventional materials management practice is no longer efficient in the context of current business perspectives. This is because the conventional practice is labour intensive, inaccurate and error prone [56]. Therefore, several authors had suggested certain industrial engineering techniques and methods to facilitate the efficiency of materials management in the construction projects. For instance, Materials Requirement Planning (MRP) is able to assist the inventory control [57, 58], Just-inTime (JIT) is the strategy for controlling the flow of construction materials [19, 23, 57, 59], and Enterprise Resource Planning (ERP) is a useful tool for integrating the flow of information in managing construction materials [59-61]. Apparently, these techniques and methods have been introduced to improve the materials management efficiency.

\section{Conclusions}

In essence this study has identified 47 influential factors that affect efficiency of materials man- agement. These factors are then categorised into 8 specific groups according to their respective themes. Initial finding shows that the highest number of influential factors is under 'management' group which consists of 15 influential factors. However, further examination will have to be conducted with practitioners to confirm the relevancy of those influential factors and each group in the context of the Malaysian construction projects. All information gained from the practitioners will then be used to develop structural modelling for materials management. Thus, it is hoped that the models will assist the construction industry players especially the contractors to improve materials management efficiency of construction projects in the near future.

\section{References}

[1] Ibrahim A.R., Roy M.H., Ahmed Z., Imtiaz G., An investigation of the status ofthe Malaysian construction industry, Benchmarking: An Int. J., 17, 4, 263270,2010 .

[2] Nwachukwu C.C., Emoh F.I., A systems approach in analysing material constraining factors to construction project management success in Nigeria, Interdisciplinary J. of Contemporary Research in Business, 2, 5, 90-105, 2010.

[3] Winch G.M., Managing construction projects - an information processing approach, (2nd ed.), West Sussex: John Wiley \& Sons, Ltd, 2010.

[4] Hughes R., Thorpe D., A review of enabling factors in construction industry productivity in an Australian environment, Construction Innovation: Information, Process, Management, 14, 2, 210-228, 2014 .

[5] Yong Y.C., Mustaffa N.E., Analysis of factors critical to construction project success in Malaysia, Engineering, Construction and Architectural Management, 19, 5, 543-556, 2012.

[6] Khan R.A., Liew M.S., Ghazali Z., Malaysian construction sector and Malaysia vision 2020: developed nation status, Procedia-Social and Behavioral Sciences, 109, 507-513, 2014.

[7] Abu Mansor S., The 7th Malaysia construction sector review and outlook seminar, http://www.kkr. gov.my/files/PRESSCIDB 3 Ogos_0.pdf, pp. 1-7, 2010.

[8] Shehu Z., Holt G.D., Endut I.R., Akintoye A., Analysis of characteristics affecting completion time for Malaysian construction projects, Built Environment Project and Asset Management, 5, 1, 52-68, 2015. 
[9] Fewings P., Construction project management - an integrated approach, (2nd ed.), Oxon. Routledge, 2012.

[10] Smith N.J., Merna T., Jobling P., Managing risk in construction projects, (3rd ed.), West Sussex: John Wiley \& Sons Ltd., 2014.

[11] Shehata M.E., El-Gohary K.M., Towards improving construction labor productivity and projects' performance, Alexandria Engineering J., 50, 4, 321-330, 2011.

[12] Meng X., The effect of relationship management on project performance in construction, Int. J. of Project Management, 30, 2, 188-198, 2012.

[13] Nagapan S., Abdul Rahman I., Asmi A., Factors contributing to physical and non-physical waste generation in construction industry, Int. J. of Advances in Applied Sciences, 1, 1, 1-10, 2012.

[14] Doloi H., Sawhney A., Iyer K.C., Rentala S., Analysing factors affecting delays in Indian construction projects, Int. J. of Project Management, 30, 4, 479-489, 2012.

[15] Abdul Rahman I., Memon A.H., Azis A., Asmi A., Abdullah N.H., Modeling causes of cost overrun in large construction projects with partial least squareSEM approach: contractor's perspective, Research J. of Applied Sciences, Engineering and Technology, 5, 6, 1963-1972, 2013.

[16] El-Gohary K.M., Aziz R.F., Factors influencing construction labor productivity in Egypt, J. of Management in Engineering, 30, 1, 1-9, 2014.

[17] Rivas R.A., Borcherding J.D., González V., Alarcón L.F., Analysis of factors influencing productivity using craftsmen questionnaires?: case study in a Chilean construction company, J. of Construction Engineering and Management, 137, 4, 312-320, 2010 .

[18] Safa M., Shahi A., Haas C.T., Hipel K.W., Supplier selection process in an integrated construction materials management model, Automation in Construction, 48, 64-73, 2014.

[19] Caldas C.H., Menches C.L., Reyes P.M., Navarro L., Vargas D.M., Materials management practices in the construction industry, Practice Periodical on Structural Design and Construction, pp. 1-8, 2014.

[20] Abdul Rahman H., Alidrisyi M.N., A perspective of material management practices in a fast developing economy: the case of Malaysia, Construction Management and Economics, 12, 5, 413-422, 1994.

[21] Kasim N., Ern P.A.S., The awareness of ICT implementation for materials management in construc- tion projects, Int. J. of Computer and Communication Technology, 2, 1, 1-10, 2010.

[22] Kasim N., ICT implementation for materials management in construction projects?: case studies, KICEM J. of Construction Engineering and Project Management, pp. 31-36, 2011.

[23] Bell L.C., Stukhart G., Attributes of materials management systems, J. of Construction Engineering and Management, 112, 1, 14-21, 1986.

[24] Chitkara K.K., Construction project management: planning, scheduling and controlling, New Delhi: Tata McGraw-Hill Publishing Company Ltd., 1998.

[25] Benton W.C., McHenry L., Construction purchasing $\&$ supply chain management, New York: The McGraw-Hill Companies, Inc., 2010.

[26] Del Pico W.J., Project control-integrating cost and schedule in construction, New Jersey: John Wiley \& Sons, Inc., 2013.

[27] Othman I., Napiah M., Potty N.S., Resource management in construction project, Applied Mechanics and Materials, 567, 607-612, 2014.

[28] Said H., El-Rayes K., Optimal material logistics planning in congested construction sites, Construction Research Congress 2012 ${ }^{\circledR}$ ASCE 2012, pp. 1580-1589, 2012.

[29] Cooke B., Williams P., Construction planning, programming and control, (3rd ed.), West Sussex: Wiley-Blackwell, 2009.

[30] Mincks W.R., Johnston H., Construction jobsite management, (3rd ed.), New York: Delmar Cengage Learning, 2011.

[31] Johnston J.E., Site control of materials - handling, storage and protection, London, Butterworths, 1981.

[32] Kazaz A., Manisali E., Ulubeyli S., Effect of basic motivational factors on construction workforce productivity in Turkey, J. of Civil Engineering and Management, 14, 2, 95-106, 2008.

[33] Kaming P.F., Olomolaiye P.O., Holt G.D., Harris F.C., Factors influencing craftsmen's productivity in Indonesia, Int. J. of Project Management, 15, 1, 2130, 1997.

[34] Jarkas A.M., Bitar C.G., Factors affecting construction labor productivity in Kuwait, J. of Construction Engineering and Management, 138, 7, 811-820, 2011.

[35] Patil A.R., Pataskar S.V., Analyzing material management techniques on construction project, Int. J. of Engineering and Innovation Tech., 3, 4, 96100, 2013. 
[36] Potty N.S., Irdus A., Ramanathan C.T., Case study and survey on time and cost overrun of multiple DESB projects, 2011 National Postgraduate Conference, pp. 1-6, 2011.

[37] Durdyev S., Mbachu J., On-site labour productivity of New Zealand construction industry?: key constraints and improvement measures, Australasian J. of Construction Economics and Building, 11, 3, 18-33, 2011.

[38] Wan S.K., Kumaraswamy M.M., Industrial management approaches for improving material control in building services works, Engineering, Construction and Architectural Management, 16, 3, 208-223, 2009 .

[39] Osmani M., Glass J., Price A.D.F., Architects' perspectives on construction waste reduction by design, Waste Management, 28, 7, 1147-1158, 2008.

[40] Toor S.U.R., Ogunlana S.O., Problems causing delays in major construction projects in Thailand, Construction Management and Economics, 26, 4, 395-408, 2008.

[41] Sambasivan M., Soon Y.W., Causes and effects of delays in Malaysian construction industry, Int. J. of Project Management, 25, 5, 517-526, 2007.

[42] Tam V.W., Shen L.Y., Fung I.W., Wang J.Y., Controlling construction waste by implementing governmental ordinances in Hong Kong, Construction Innovation, 7, 2, 149-166, 2007.

[43] Enshassi A., Mohamed S., Abu Mustafa Z., Mayer P.E., Factors affecting labour productivity in building projects in the Gaza strip, J. of Civil Engineering and Management, 13, 4, 245-254, 2007.

[44] Navon R., Berkovich O., Automated materials management and control model, Construction Research Congress 2005, pp. 287-295, 2005.

[45] Assaf S.A., Al-Hejji S., Causes of delay in large construction projects, Int. J. of Project Management, 24, 4, 349-357, 2006.

[46] Osmani M., Glass J., Price A.D., Architect and contractor attitudes to waste minimization, Proceeding of the Institution of Civil Engineers: Waste and Resource Management, 159, 65-72, 2006.

[47] Abdul Kadir M.R., Lee W.P., Jaafar M.S., Sapuan S.M., Ali A., Factors affecting construction labour productivity for Malaysian residential projects, Structural Survey, 23, 1, 42-54, 2005.

[48] Ekanayake L.L., Ofori G., Building waste assessment score: design-based tool, Building and Environment, 39, 7, 851-861, 2004.
[49] Manavazhi M.R., Adhikari D.K., Material and equipment procurement delays in highway projects in Nepal, Int. J. of Project Management, 20, 8, 627632, 2002.

[50] Odeh A.M., Battaineh H.T., Causes of construction delay: traditional contracts, Int. J. of Project Management, 20, 1, 67-73, 2002.

[51] Makulsawatudom A., Emsley M., Factors affecting the productivity of the construction industry in Thailand: the project managers' perception, 17th Annual ARCOM Conference, 1, 281-290, 2001.

[52] Zakeri M., Olomolaiye P.O., Holt G.D., Harris F.C., A survey of constraints on Iranian construction operatives' productivity, Construction Management and Economics, 14, 5, 417-426, 1996.

[53] Ogunlana S.O., Promkuntong K., Jearkjirm V., Construction delays in a fast-growing economy: comparing Thailand with other economies, Int. J. of Project Management, 14, 1, 37-45, 1996.

[54] Abdul Majid M., McCaffer R., Factors of nonexcusable delays that influence contractors' performance, J. of Management in Engineering, 14, 3, 4249, 1998.

[55] Guo J., Construction materials management system design based on barcode, Advanced Materials Research, 860, 2825-2829, 2014.

[56] Dey P.K., Re-engineering materials management: a case study on an Indian refinery, Business Process Management Journal, 7, 5, 394-408, 2001.

[57] Ibn-homaid N.T., A comparative evaluation of construction and manufacturing materials management, Int. J. of Project Management, 20, 4, 263-270, 2002.

[58] Barriga E., Jeong J., Material requirements planning for a manufactured housing facility, J. of Architectural Engineering, 11, 3, 91-98, 2005.

[59] Akintoye A., Just-in-Time application and implementation for building material management, Construction Management and Economics, 13, 2, 105113, 1995.

[60] Fawcett S., Birou L., Just-in-time sourcing techniques: current state of adoption and performance benefits, Production and Inventory Management J., 34, 18-18, 1993.

[61] Ng S.T., Shi J., Fang Y., Enhancing the logistics of construction materials through activity-based simulation approach, Engineering, Construction and Architectural Management, 16, 3, 224-237, 2009. 